“ (C) 2017 IEEE. Personal use of this material is permitted. Permission from IEEE must be obtained for all other uses, in any current or future media, including

reprinting/republishing this material for advertising or promotional purposes, creating new collective works, for resale or redistribution to servers or lists, or reuse of any copyrighted component of this work in other works." 


\title{
Detection of Gait Initiation Failure in Parkinson's disease based on Wavelet Transform and Support Vector Machine
}

\author{
Quynh Tran Ly ${ }^{\mathrm{a}}$, Student Member, IEEE, A.M. Ardi Handojoseno ${ }^{\mathrm{a}, \mathrm{c}, \dagger}$, Moran Gilat ${ }^{\mathrm{b}}$, Rifai Chai ${ }^{\mathrm{a}}$, \\ Kaylena A. Ehgoetz Martens ${ }^{\mathrm{b}}$, Matthew Georgiades ${ }^{\mathrm{b}}$, Member, IEEE, Ganesh R. Naik $^{\mathrm{a}}$, Yvonne Tran ${ }^{\mathrm{a}}$,

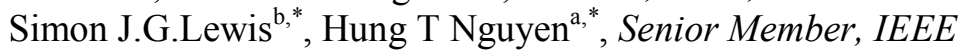

\begin{abstract}
Gait initiation Failure (GIF) is the situation in which patients with Parkinson's disease (PD) feel as if their feet get "stuck" to the floor when initiating their first steps. GIF is a subtype of Freezing of Gait (FOG) and often leads to falls and related injuries. Understanding of neurobiological mechanisms underlying GIF has been limited by difficulties in eliciting and objectively characterizing such gait phenomena in the clinical setting. Studies investigating the effects of GIF on brain activity using EEG offer the potential to study such behavior. In this preliminary study, we present a novel methodology where wavelet transform was used for feature extraction and Support Vector Machine for classifying GIF events in five patients with PD and FOG. To deal with the large amount of EEG data, a Principal Component Analysis (PCA) was applied to reduce the data dimension from 15 EEG channels into 6 principal components (PCs), retaining 93\% of the information. Independent Component Analysis using Entropy Bound Minimization (ICA-EBM) was applied to 6 PCs for source separation with the aim of improving detection ability of GIF events as compared to the normal initiation of gait (Good Starts). The results of this analysis demonstrated the correct identification of GIF episodes with an $83.1 \%$ sensitivity, $89.5 \%$ specificity and $86.3 \%$ accuracy. These results suggest that our proposed methodology is a promising non-invasive approach to improve GIF detection in PD and FOG.
\end{abstract}

\section{INTRODUCTION}

Freezing of gait (FOG) is defined as a brief, episodic absence or marked reduction of forward progression of the feet despite the intention to walk [1]. In our Timed Up and Go experiment, different types of FOG were distinguished relating to the clinical situation in which they occurred, such as gait initiation failure (GIF), freezing whilst passing through a narrow space, freezing whilst dual-tasking, freezing on an open runway and freezing during turning [1]. GIF is a very common situation in which patients with PD experience FOG when initiating their first step. The prevalence of GIF ranges from $21 \%$ to $24 \%$ of all witnessed FOG in PD $[1,2]$. Behavioral manifestations during GIF can be observed like as trembling in place, shuffling with minimal forward movement, or complete akinesia [1]. Gait Initiation is a complex task which requires both motor and

\footnotetext{
${ }^{a}$ Faculty of Engineering and Information Technology, University of Technology, Sydney, Broadway, NSW 2007, Australia

b Parkinson's Disease Research Clinic, Brain and Mind Center, University of Sydney, Level 4, Building F, 94 Mallet Street, Camper down, NSW, 2050, Australia.

${ }^{c}$ Faculty of Science and Engineering, Sanata Dharma University, Paingan, Sleman, Yogyakarta, 55281, Indonesia.

* Dual senior authorship.

${ }^{\dagger}$ Deceased April 8, 2017.
}

cognitive processing to enable the correct selection, timing and scaling of movements [3]. From a functional perspective, GIF is clinically important as a trigger for FOG because initiating gait is frequently attempted every day. The increased risk of falls induced by GIF negatively affects the quality of life for patients with PD and can result in injury as well as nursing home placement.

In recent years, a number of researches have reported the detection of FOG based on the leg oscillations using accelerometer, gyroscope and goniometers. However, using such sensors based FOG detection systems could not identify GIF periods which are not associated with the trembling of the legs. Our group previously studied the brain activity underlying GIF by analyzing energy power of surface ambulatory EEG signals underlying this event [2, 4]. However, as data was derived from a large number of channels, computational complexity was increased for performing further signal processing methods. With the goal of developing a faster and better classification system, the current paper sought to analyze EEG features to detect GIF using principal component analysis (PCA) for data dimensional reduction, Independent Component Analysis with Entropy Bound Minimization (ICA-EBM) for source separation, Wavelet Transform (WT) decomposition for feature extraction and Support Vector Machine (SVM) for classification. PCA is used to transform a high dimensional EEG dataset to a low dimensional orthogonal feature set while retaining the maximum information of the original high dimensional dataset [5]. ICA-EBM was developed to separate the EEG data source to improve the classification [6].

The main contributions of this paper are the novel techniques applied, in which PCA is used for reducing data dimension, ICA-EBM for source separation, WT decomposition for feature extraction and SVM for classification; which has not been explored previously to detect GIF in PD. These results suggest that our proposed methodology is a promising non-invasive approach for improvement of GIF detection.

\section{METHODS}

\section{A. Data Collection and Pre-processing}

EEG data was obtained from five Parkinson's disease patients who were recruited from the Parkinson's disease Research Clinic at the Brain and Mind Center, The University of Sydney. This study was approved for by The Human Research and Ethics Committee, University of Sydney. They were tested in their practically-defined 'off' medication state, following overnight (minimum 12 hours 
since last dose) withdrawal of dopaminergic therapy. The subjects demonstrated multiple episodes of GIF ( $40 \%$ of gait initiations elicited a GIF) during a structured series of Timed Up and Go tasks. Good Starts (GS) is defined as the 2-second period after the patients were able to effectively initiate the first step. GIF is defined as the period when the patients tried to take a first steps but failed to do so. Both were determined according to the time onset and offset as scored on the video during the TUG tasks.

Based on our previous FOG findings, EEG data was acquired from 15 electrodes using a Bio semi Active Two system [4]. These electrodes positioned over the following cortical regions: frontal F3, F4, FC1, FC2 (motor planning and working memory), central C3, C4, CP1, CP2, CZ (motor execution), parietal P3, PZ, P4 (sensory integration) and occipital O1, OZ, O2 (visual area). References signal was taken by averaging 2 electrodes placed on the ear lobes. The recording was segmented to 1 -second durations and digitized at $512 \mathrm{~Hz}$.

In this study, 66 seconds of EEG data samples of GS were matched to 66 seconds of data samples of GIF as recorded from five PD patients. Data samples were then filtered using band-pass filter at $0.5-50 \mathrm{~Hz}$. Eye movement and heart rate signals artifacts were eliminated using Automatic Artifact Removal (AAR) in the EEGLab from all electrode locations of raw EEG data [4].

\section{B. Feature Extraction}

Due to the strengths in time-frequency method, WT was chosen to extract the EEG data. WT is defined as the convolution between the signal and wavelet function generated by dilations and translation of scaled mother wavelet [7]. In this research, the discrete wavelet transforms (DWT) based on dyadic scales and positions is used [7]. This is computed as

$$
D W T(j, k)=\frac{1}{\sqrt{\left|2^{j}\right|}} \int_{-\infty}^{\infty} x(t) \Psi\left(\frac{t-2^{j} k}{2^{j}}\right) d t
$$

where $2^{j}$ denotes the scale and $2^{j} k$ denotes the time localization and $\Psi$ denotes the mother wavelet function.

The wavelet decomposition for an EEG signal $x(t)$ at scales $j$, time point $k$ could be calculated as:

$$
x(t)=\sum_{k=-\infty}^{\infty} A(k) \varphi_{J, k}(t)+\sum_{j=0}^{\infty} \sum_{k=-\infty}^{\infty} D(j, k) \Psi_{j, k}(t)
$$

where $A(k), D(j, k), \varphi_{J, k}, \Psi_{j, k}(t)$ denotes approximation coefficients, detail coefficients, scaling function, and wavelet functions, respectively.

Daubechies wavelet of order $4(\mathrm{db} 4)$ were selected as the wavelet function since its smoothing feature has been confirmed to work well in discovering changes of EEG signals [7]. For EEG sampled at $512 \mathrm{~Hz}$, a six level decomposition ( $11, \mathrm{~d} 2, \mathrm{~d} 3, \mathrm{~d} 4, \mathrm{~d} 5, \mathrm{~d} 6)$ were computed by squaring and summing the wavelet coefficients of the decomposed level. Four levels of coefficients were corresponded approximately to our four EEG sub-band, d6 (theta: 4-8 Hz), d5 (alpha: 8-13 Hz), d4 (low beta: 13-21 Hz), d3 (high beta: 21-38 Hz); which were used for further analysis. In order to eliminate differences between electrodes and individual subjects, a Z-transformation was applied to normalize EEG data.

\section{Principal Component Analysis (PCA) for dimensional reduction}

The computational complexity increases with a higher dimensional dataset. Therefore, PCA was applied to decrease the dimension dataset into a lower dimension dataset that still covers enough information $[5,9]$.

Given a raw EEG dataset $x_{t}$ and $\sum_{k=1}^{M} x_{k}$, with $M$ denoting the number of EEG samples, the covariance matrix $C$ of PCA was computed as:

$$
C=\frac{1}{M} \sum_{j=1}^{M} x_{j} x_{j}^{T}
$$

where $x_{j} x_{j}^{T}$ denotes a vector of $N \times N$ matrix, with $N$ denoting the number of EEG channels. PCA calculated the eigenvector as

$$
\lambda \mu=C \mu
$$

where $\mu$ denotes the eigenvectors of $C$ and $\lambda$ denoted the eigenvalues. The principal component of $S_{t}$ as the orthogonal transformation of $x_{t}$ is calculated as followings

$$
S_{t}=\mu^{T} x_{t}
$$

Principal components contain the maximum variance in the data. The origin of the new coordinate system is located in the center of the data points. The first PC points in the direction of highest variance, the second PC points in the direction of second highest variance and so on. The variances captured for the corresponding PCs were calculated as well. The eigenvectors are ranked in a descending order of eigenvalues. By choosing only the first few eigenvectors, PCA performed a dimension reduction from high dimensional EEG data into low dimensional feature containing only a few principle components, but that still contain enough information of the original data.

\section{Source separation using ICA-EBM}

Next, the ICA-EBM was used to separate sources. ICA is one of the Blind Source Separation algorithms. It worked by separating the mixed information into independent components [6]. ICA-EBM provides flexible density matching through the use of contrast functions based on the maximum entropy principle. ICA-EBM separated both subor super-Gaussian mixtures using only a small class of nonlinear functions. After pre-processing using PCA and ICA-EBM, the 6 PCA and ICA separated data were extracted in the form of wavelet transform and fed into our classifier.

\section{E. Classification}

The significant maximum ( $A^{\max }$ ) and mean value of wavelet decomposition of 4 sub-bands from each electrode taken from two events were chosen as the features to detect GIF. We utilized Support Vector Machine (SVM) to detect GIF because of its accuracy and ability to deal with a large number of predictors [8]. The significant features were divided into half portions, one for the training set and the same amount for the testing set. SVM with a Radial Basis Function (RBF) kernel is computed as follows [8]. 


$$
R B F_{J S}\left(x, \mu_{k}, \sigma_{k}\right)=\exp \left(-\frac{J S\left(x|| \mu_{k}\right)}{2 \sigma_{k}^{2}}\right)
$$

where $x, k, \mu_{k}$ and $\sigma_{k}$ denote a random vector, support vector index, support vector centroid and support vector radius, respectively. $J S$ denotes the Jensen-Shannon (JS) divergence between $x$ and $\mu_{k}$.

The $J S$ divergence is a symmetrized and smoothed version of the Kullback-Leibler $(K L)$ divergence. $J S$ divergence is calculated as

$$
J S(P \| Q)=\frac{1}{2} K L(P \| M)+\frac{1}{2} K L(Q \| M)
$$

where $\mathrm{P}, \mathrm{Q}$ denote discrete probability distribution, $M=(P+Q) / 2$ denotes the central probability mass function and $K L$ divergence is computed as

$$
K L(R \| S)=\sum_{x} R(x) \log \frac{R(x)}{S(x)}
$$

A particle swarm ensemble clustering algorithm known as Ensemble Rapid Centroid Estimation (ERCE) was used for estimating the parameters for the RBF kernel [8]. When using ERCE, $\mu_{\mathrm{k}}$ and $\sigma_{k}$ could be inferred from the training data by using the following four steps:

1. Execute ERCE to cluster the training set to an arbitrary number based on $J S$ distance.

2. Aggregate the ensemble clustering results using average linkage to get the final clustered sets $\mathbb{C}_{k}$. The corresponding centroid vector $\mu_{k}$ was computed as:

$$
\mu_{k}=E\left[x \mid \mathbb{C}_{k}\right]=\frac{1}{\left|\mathbb{C}_{k}\right|} \sum_{x \in \mathbb{C}_{k}} x
$$

3. The RBF kernel radius $\sigma_{k}$ was taken as the square root of conditional $J S$ divergence, which is given as follows

$$
\sigma_{k}^{2}=E\left[J S\left(x|| \mu_{k}\right) \mathbb{C}_{k}\right]=\frac{1}{\left|\mathbb{C}_{k}\right|} \sum_{x \in \mathbb{C}_{k}} J S\left(x|| \mu_{k}\right)
$$

4. The SVM was then trained using the LS algorithm

\section{RESULTS}

\section{A. Feature Extraction Results}

Figure 1 shows the time-frequency distributions in two events GS and GIF from one PD patients using EEGLab toolbox. In this analysis, we explored the mean event-related changes in spectral power at each time during the epoch and at each frequency in such PD patient. It is observed that significant increase in power occurred at low beta $(13-21 \mathrm{~Hz})$

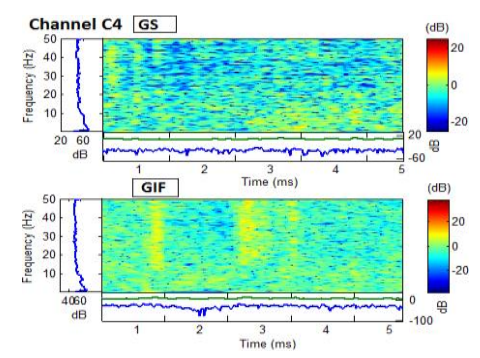

Figure 1. Time-frequency distribution for GS and GIF of motor cortex C4.
TABLE I. FEATURE EXTRACTION

\begin{tabular}{ccccc}
\hline & Band & GS & GIF & Significant \\
\hline & & $\left(A^{\text {max }} \pm \mathrm{std}\right) * 0.1$ & $\left(A^{\text {max }} \pm \mathrm{std}\right) * 0.1$ & \\
\hline $\mathrm{F} 3$ & $\mathrm{~h} \beta$ & $0.068 \pm 0.072$ & $0.076 \pm 0.061$ & $*$ \\
$\mathrm{~F} 4$ & $\alpha$ & $0.132 \pm 0.067$ & $0.113 \pm 0.092$ & $*$ \\
\hline $\mathrm{C} 3$ & $\mathrm{~h} \beta$ & $0.091 \pm 0.079$ & $0.10 \pm 0.065$ & $*$ \\
$\mathrm{C} 4$ & $\mathrm{~h} \beta$ & $0.091 \pm 0.079$ & $0.107 \pm 0.065$ & $*$ \\
$\mathrm{CP} 1$ & $\mathrm{~h} \beta$ & $0.088 \pm 0.072$ & $0.119 \pm 0.066$ & $* *$ \\
$\mathrm{CP} 2$ & $\mathrm{~h} \beta$ & $0.088 \pm 0.072$ & $0.119 \pm 0.079$ & $*$ \\
\hline $\mathrm{P} 3$ & $\theta$ & $0.359 \pm 0.202$ & $0.377 \pm 0.158$ & $*$ \\
& $\boldsymbol{h} \boldsymbol{\beta}$ & $\mathbf{0 . 1 1 2} \pm \mathbf{0 . 0 7 5}$ & $\mathbf{0 . 1 5 0} \pm \mathbf{0 . 0 7 7}$ & $* *$ \\
$\mathrm{PZ}$ & $\mathrm{h} \beta$ & $0.114 \pm 0.077$ & $0.150 \pm 0.093$ & $*$ \\
$\boldsymbol{P 4}$ & $\boldsymbol{h} \boldsymbol{\beta}$ & $\mathbf{0 . 0 8 4} \pm \mathbf{0 . 0 6 1}$ & $\mathbf{0 . 1 1 5} \pm \mathbf{0 . 0 5 5}$ & $* *$ \\
\hline $\boldsymbol{O 1}$ & $\boldsymbol{h} \boldsymbol{\beta}$ & $\mathbf{0 . 2 0 3} \pm \mathbf{0 . 0 9 7}$ & $\mathbf{0 . 2 5 2} \pm \mathbf{0 . 0 9 6}$ & $* *$ \\
$\boldsymbol{O 2} 2$ & $\mathrm{1} \boldsymbol{0 . 0 5 7}$ & $0.197 \pm 0.115$ & $0.224 \pm 0.097$ & $*$ \\
& $\boldsymbol{h} \boldsymbol{\beta}$ & $\mathbf{0 . 1 9 9} \pm \mathbf{0 . 0 9 5}$ & $\mathbf{0 . 2 4 2} \pm \mathbf{0 . 0 9 3}$ & $* *$ \\
\hline
\end{tabular}

*: $\quad$ Significant at $p \leq 0.05$ and Cohen's $d<0.4$

**. Significant at $p \leq 0.001$ and Cohen's $d \geq 0.4$

and high beta (21-38 Hz) frequency bands during GIF.

For the feature extraction, 132 seconds from the two events GS and GIF (66 seconds each) from all 5 PD patients were analyzed. Table I shows the significant maximum amplitude from 15 locations based on four EEG frequency bands activities. A Wilcoxon signed-rank test with $p$-value $\leq$ 0.001 and a Cohen's effect size $d \geq 0.4$ were used to investigate significant differences between periods of GS and GIF. A decrease in alpha was found in F4 and an increase in theta was found in P3. However, overall it is apparent that increases in high beta frequency throughout the cortical brain regions underlie GIF compared to Good starts. More specifically, in the context of GIF, high beta activity experienced the largest significant rise in parietal regions P3 $(\mathrm{d}=0.5013), \quad \mathrm{P} 4 \quad(\mathrm{~d}=0.5378)$ and occipital regions $\mathrm{O} 1$ $(\mathrm{d}=0.499), \mathrm{O} 2$ ( $\mathrm{d}=0.4577)$. This finding is similar to our previous studies using PSD for analysis [2, 4].

\section{B. PCA, ICA-EBM and input for classification}

The collected EEG dataset comprised of a matrix with dimension of $15 \times 66 \times 512$ (number of EEG channels $\times 66$ seconds $\times 512$ data points) for both GIF and GS. These matrices were fed into the PCA process to reduce the data dimension. The PCA transformed the original coordinate system into new coordinates called principal components (PCs). Eigenvalues values are shown in a scree plot (see Figure 2), which presents percent variance captured versus number of principal components. It can be seen that with 6 PCs, it already covered more than $93 \%$ of the variance of the original of 15 EEG channels. PC 1 accounted for $64.08 \%$ of the total variation in the data and PC 2 accounted for $15.07 \%$ of the total variation in the data. This study applied a threshold of $93 \%$, which generated orthogonal transformation from $\mathrm{PC} 1$ to $\mathrm{PC} 6$, to be used for further processing.

After applying the PCA, the original high dimension of the EEG dataset was reduced from $15 \times 66 \times 512$ (number of EEG channels $\times$ second of data $\times$ data point per second)

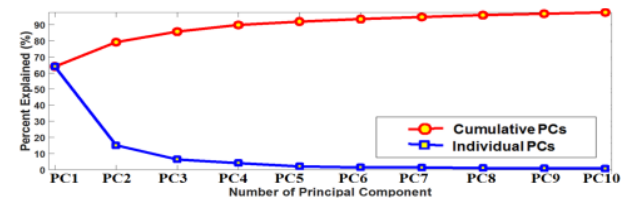

Figure 2. Scree plot of the principal components 
to $6 \times 66 \times 512$ (number of PCs $\times$ second of data $\times$ data point per second) for GIF and a similar matrix for GS stages. Such a reduced dataset dimension resulted in a lower computational complexity; here-after called the 6 PCs EEG data. Next, the 6 PCs EEG data was fed to the ICA-EBM, resulted in the 6-channels ICA separated sources. These optimized sources were further segmented for feature extraction using wavelet transform. In order to build a faster and better classification system, only significant statistical different features between two groups of data ( $p$-values $<$ $0.05)$ were chosen as input for the GIF detection.

\section{Classification results}

For comparison purposes, the classification result from the original 15 EEG channels without the use of PCA is also reported. Table II shows the classification result for GIF detection. The classification (with PCA) using 6 PCs EEG data resulted in a sensitivity of $80.6 \%$, a specificity of $78.9 \%$ and an accuracy of $79.7 \%$, while the classification using original 15 EEG channels (without PCA) resulted in a sensitivity of $80.7 \%$, a specificity of $80.6 \%$ and an accuracy of $80.6 \%$. The results thus confirmed that the PCA successfully reduced the dimension of the EEG data, provided similar accuracy (slightly improved) accuracy as compared to the original dataset (without PCA) for GIF detection. Further improvements were obtained when using the combination of ICA-EBM as the source separator is used. These results (with PCA, ICA-EBM) achieved a good sensitivity of $83.1 \%$, specificity of $89.5 \%$ and an accuracy of $86.3 \%$ for detecting GIF.

\section{DISCUSSION}

In this paper we compared ambulatory EEG during Gait Initiation Failure and Good Starts in patients with PD and who had FOG. The fast temporal changes occurring in the brain during GIF were found to be associated with an overall increase in high beta activities over frontal, central, parietal and occipital cortical locations. FOG has previously been associated with high beta oscillations in the subthalamic nucleus, which cohere with both supplementary and primary motor areas [3]. Together these may constitute inhibitory control over motor actions when response conflict arises [3]. This could explain the high beta frequencies found in frontal and central cortical regions during GIF in the current study. High beta oscillation in parietal and occipital locations support the notion that PD patients with GIF suffered from impaired sensory integration and thus had to gain more information from the environment to initiate gait [3].

The classification results indicated that the use of PCA for data dimension reduction, ICA-EBM for source separation, wavelet for feature extraction and Support Vector Machine for classifier achieved the best performance indicators for GIF detection, with sensitivity increasing by $2.4 \%$ (from $80.7 \%$ to $83.1 \%$ ) and accuracy increasing by $5.7 \%$ (from $80.6 \%$ to $86.3 \%$ ) as compared with the case without using PCA and ICA-EBM. This finding therefore suggests that PCA was successfully applied for data dimensionality reduction, but still retained sufficient information of the original data which are benefits for solving big data dimension problems in such EEG studies [9].
TABLE II. CLASSIFCATION RESULTS

\begin{tabular}{ccccccccc}
\hline PCA & ICA-EBM & Dimension & \multicolumn{3}{c}{ Training } & \multicolumn{3}{c}{ Testing } \\
\hline & & & Sen & Spe & Acc & Sen & Spe & Acc \\
\hline No & No & 15 channels & 81.0 & 80.8 & 80.9 & 80.7 & 80.6 & 80.6 \\
\hline Yes & No & 6 PCs & 81.0 & 79.3 & 80.1 & 80.6 & 78.9 & 79.7 \\
\hline Yes & Yes & 6 PCs & $\mathbf{8 3 . 4}$ & $\mathbf{8 9 . 7}$ & $\mathbf{8 6 . 6}$ & $\mathbf{8 3 . 1}$ & $\mathbf{8 9 . 5}$ & $\mathbf{8 6 . 3}$ \\
\hline
\end{tabular}

Sen: Sensitivity; Spe: Specificty; Acc: Accuracy

\section{CONCLUSION}

We have successfully used EEG signals to investigate brain dynamic changes underlying gait initiation failure in PD and detected these events with high performance. The preliminary study provides optimism for the development of a real-time device for GIF detection that could be employed in everyday walking situations in PD patients. Further research should focus on optimizing the above techniques for a wider pool of participants and also investigate the efficacy of GIF detection system in real time. It is hoped that an increased understanding of underlying neurobiology will ultimately promote the development of novel therapies and technologies to assist the management of FOG in PD.

\section{ACKNOWLEDGMENT}

The authors would like to dedicate this work to the memory of Dr A.M. Ardi Handojoseno who contributed significantly to our research in Parkinson's disease. His intellect, kindness and compassion will always remain deeply in our hearts.

\section{REFERENCES}

[1] J. D. Schaafsma, Y. Balash, T. Gurevich, A. L. Bartels, J. M. Hausdorff, and N. Giladi, "Characterization of freezing of gait subtypes and the response of each to levodopa in Parkinson's disease," European Journal of Neurology, vol. 10, pp. 391-398, 2003

[2] A. M. Ardi Handojoseno, J. M. Shine, T. N. Nguyen, Y. Tran, S. J. G. Lewis, and H. T. Nguyen, "Analysis and Prediction of the Freezing of Gait Using EEG Brain Dynamics," Neural Systems and Rehabilitation Engineering, IEEE Transactions on, vol. 23, pp. 887-896, 2015

[3] M. J. Georgiades, M. Gilat, K. A. Ehgoetz Martens, C. C. Walton, P. G. Bissett, J. M. Shine, et al., "Investigating motor initiation and inhibition deficits in patients with Parkinson's disease and freezing of gait using a virtual reality paradigm," Neuroscience, vol. 337, pp. 153162,2016

[4] Q. T. Ly, A. M. A. Handojoseno, M. Gilat, N. Nguyen, R. Chai, Y. Tran, H. T. Nguyen et al., "Identifying montages that best detect the electroencephalogram power spectrum alteration during freezing of gait in Parkinson's disease patients," in 2016 38th Annual International Conference of the IEEE Engineering in Medicine and Biology Society (EMBC), pp. 6094-6097, 2016

[5] U. R. Acharya, S. V. Sree, A. P. C. Alvin, and J. S. Suri, "Use of principal component analysis for automatic classification of epileptic EEG activities in wavelet framework," Expert Systems with Applications, vol. 39, pp. 9072-9078, 2012

[6] X. L. Li and T. Adali, "Independent Component Analysis by Entropy Bound Minimization," IEEE Transactions on Signal Processing, vol. 58, pp. 5151-5164, 2010

[7] C.S. Burrus, R.A. Gopinath, and H.Guo, Introduction to wavelets and wavelet transforms: a primer, New Jersey: Prentice-Hall,pp.2-7, 1998.

[8] M. Yuwono, S. Su, B. Moulton, Y. Guo, and H. Nguyen, "An algorithm for scalable clustering: Ensemble rapid centroid estimation," in Evolutionary Computation (CEC), 2014 IEEE Congress on, pp. 1250-1257, 2014

[9] R. Chai, G. Naik, T. N. Nguyen, S. Ling, Y. Tran, A. Craig, et al., "Driver Fatigue Classification with Independent Component by Entropy Rate Bound Minimization Analysis in EEG-based System” 